\title{
Impact of granular slugs on rigid targets: effect of grain shape and fracture
}

\author{
A. Goel ${ }^{\mathrm{a}}$, H.N.G. Wadley ${ }^{\mathrm{b}}$ and V.S. Deshpande $\mathrm{a}^{\mathrm{a}^{*}}$ \\ a Department of Engineering, University of Cambridge, \\ Trumpington Street, Cambridge CB2 1PZ, UK. \\ ${ }^{\mathrm{b}}$ Department of Material Science \& Engineering, School of Engineering and Applied Science, \\ University of Virginia, Charlottesville, VA 22904, USA.
}

\begin{abstract}
The effect of grain shape and fracture on the interaction of high velocity granular slugs with rigid stationary targets is analysed for targets in normal and inclined orientations. The granular slugs comprise spherical, rod-shaped or cubic grains and are constructed by connecting together spherical sub-particles with either rigid or beam connectors. The case when grain fracture is suppressed (rigid connectors between sub-particles) is first analysed. With increasing grain aspect ratio, the grains tend to slide rather than roll on the target surface and this increases frictional interactions with the target surface. However, these enhanced frictional forces do not affect the momentum transmitted into normally oriented targets due to the symmetry of the problem. By contrast, the break in the symmetry for inclined targets results in the transmitted momentum increasing with grain aspect ratio. Fracture of the grains (as modelled by the fracture of the beam connectors between sub-particles) is shown to affect the momentum transmitted into the inclined targets. This is a consequence of fracture resulting in a change in grain shape. In this case the simulations show that the transmitted momentum is a function of the initial grain shape, the fracture properties of the grains and the impact velocity. In fact, grain fracture results in an enhanced transmitted momentum for initially cubic grains but fracture of grains with a high initial aspect ratio results in a reduction in transmitted momentum as these grains fragment into more spherically shaped grains.
\end{abstract}

Keywords: fluid-structure interaction, granular media, fracture, discrete element method.

*Corresponding author. E-mail address: vsd@eng.cam.ac.uk. 


\section{Introduction}

Determining the loads applied to structures by the impact of high velocity granular matter is a problem of significant scientific research and technological interest. For example, the design of ground vehicles subjected to landmine explosions depends upon a detailed understanding of the forces applied during the interaction of the vehicle structure with explosively accelerated soil. The phenomena that contribute to this dynamic loading following detonation of shallowburied explosives are very complex and current understanding is largely based on empirical models that estimate the impulsive loads imposed by soil ejecta (Westine et al., 1985). Such models have helped inform more recent experimental characterizations of buried explosive events (Bergeron et al., 1998; Neuberger et al., 2007a). However, there still remains significant uncertainty especially on how properties of the soil affect the loads imposed on structures by the high velocity granular media.

Reduced-scale experiments have been increasingly used for parametric studies to understand phenomena associated with dynamic loading of structures by explosively accelerated granular media. For example, Hlady (2004) employed $0.025 \mathrm{~kg}$ buried charges in conjunction with a vertical impulse pendulum to investigate the influence of soil properties. Increasing the water saturation was shown to increase the momentum imparted to the target plate, but only if a threshold moisture content was exceeded. In a joint study, Taylor et al. (2005) and Fourney et al. (2005) considered the scalability of buried charge experiments and demonstrated an influence of plate mass. This dependence on plate mass indicates that fluid-structure interaction (FSI) effects may play a role for granular media impacting structures. Neuberger et al. (2007a, 2007b) examined the deformation of clamped plates loaded by spherical charges either flush buried in dry sand (i.e. zero sand overburden) or in air alone and showed that the presence of sand increases the deformation of the target plate.

In the majority of experimental studies, control of the soil conditions is relatively poor and this results in a scatter of $\pm 15 \%$ from nominally identical tests (Clarke et al., 2014). In fact, such dispersion in observations persists even when soil as specified by the NATO standardisation agreement STANAG 4569 (NATO, 2014) is used and the scatter in observations is commonly attributed to variability in the granular media. For example, Rigby et al. (2017) have shown that particle size distributions and soil type (sand versus clay versus gravel) strongly influences the loading imposed on structures. Similarly, Ehrgott et al. (2011) conducted a series of experiments with both surface and shallow buried test charges where they carefully varied the soil type and demonstrated a significant variation in impulse loads with soil properties. They attributed the dependence on the soil type to differences in the generated soil ejecta rather than the efficiency of momentum transfer during particle-target impact. An alternative approach to better control the experimental conditions was developed by Dharmasena et al. (2013) and involves explosively accelerating silica glass microspheres that encase a spherical explosive charge. This approach has been utilised in a number of studies including by Kyner et al. (2017) as it gives repeatable results and therefore is more suitable for detailed numerical modelling. However, the drawback of this approach is that that the influence of soil type, including particle shape, upon the impact loading are neglected.

A number of numerical approaches have been proposed to model the complex dynamic interaction of high velocity granular media with structures. For example, Rimoli et al. (2011) used the soil constitutive model of Deshpande et al. (2009) to deduce the impulse applied to structures by explosively accelerated spherical sand shells. Grujicic et al. (2008a, 2008b, 2006) and Wang et al. (2004) used coupled Eulerian/Lagrangian simulations of landmine explosions, and attempted to compare their predictions with blast impulse and plate deformation 
measurements from Bergeron and Tremblay (2000) and Foedinger (2005). Recently, coupled discrete particle/continuum simulations have been increasingly used to investigate the response of structures impacted by high velocity granular media. For example, Borvik et al. (2011) followed by Dharmasena et al. (2013) and Holloman et al. (2015a, 2015b) used this approach to simulate the response of a variety of monolithic and sandwich structures loaded by high velocity granular sprays generated by the explosive detonations. These discrete particle approaches typically use spheres to represent the granular particles although some differences exist in individual implementations. For example, Liu et al. (2013) and Dharmasena et al. (2013) allow for three rotational and three translational degrees of freedom for the spherical particles while the approaches of Borvik et al. (2011) and Holloman et al. (2015a, 2015b) restrict rotational motion of the spheres, even though tangential frictional forces that impose torques on the particles are included in the analyses. Since all these studies were restricted to spherical particles, they were unable to investigate the effect of particle angularity on the dynamic flow and impact of granular media. However, the static and dynamic flow of granular media is well-known to be strongly influenced by the shape of the granular particles. For example, Cho et al. (2006) observed that particle angularity hinders particle rotation, while Farhadi and Behringer (2014) showed that particle shape significantly influenced the jamming behavior of granular media. It is worth emphasizing here that particle angularity may not arise only from the initial particle shape; it can also develop by particle fracture during impact events. In fact, a number of studies (Behera et al., 2005; Cil and Alshibli, 2012; Parab et al., 2014; Zheng et al., 2015) have analyzed the fracture of particles including its effect in the context of sand and grit blasting, the change in particle shape and its effect on the interaction of granular media with a structure are not well studied or understood. Similar issues are also encountered during impact of water droplets on surfaces in the context of waterjet peening (Xie and Rittel, 2017).

Loading of targets by high velocity granular media generated by explosive detonations typically results in poorly characterized granular sprays. This is because the explosive gases obscure the view and only the outer surface of granular front is visible; see for example Pickering et al. (2012) and Dharmesena et al. (2013). Park et al. (2013) developed a technique to generate a high-velocity granular slug within a laboratory setting and without the need for the detonation of an explosive. They thus circumvented the problems associated with explosive detonations. Uth and Deshpande (2014) and Uth et al. (2015) employed this setup to investigate the dynamic response of monolithic and sandwich structures impacted by such granular slugs. These measurements indicated that while the momentum transmitted into the inclined targets was less than that for the normally oriented counterparts, it was significantly higher than that anticipated from analyses which neglect frictional interactions between the granular particles and the target; see for example Cheng et al. (2007) as well as Johnson and Gray (2011). Goel et al. (2017) subsequently showed that frictional interactions between the particles of granular medium and the target surface significantly increase the momentum transfer to the target. Moreover, Goel et al. (2017) demonstrated that discrete particle numerical simulations using spherical particles were unable to capture these frictional effects. They argued that modelling the angular particle shapes is critical in order to accurately predict the interaction of high velocity granular media with a target. The primary aim of this study is to begin a fundamental investigation of the effect of particle shape on the momentum transmitted to structures by the impact of a granular media. We numerically analyze the prototypical problem of the impact of granular slugs with rigid stationary targets at normal and inclined angles of incidence as shown in Fig. 1, and investigate the effect of particle shape and particle fracture on the momentum transferred from the granular slug into the targets. 
(a)
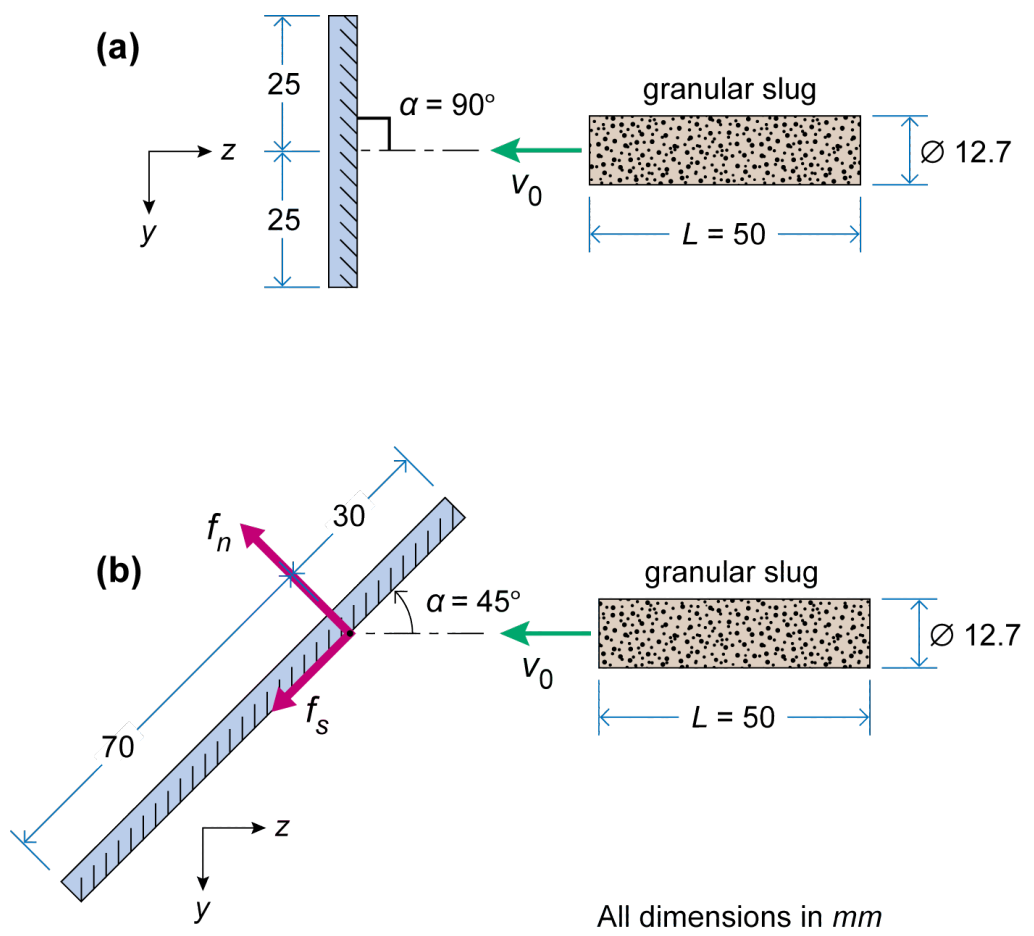

Figure 1: Impact of cylindrical granular slugs with rigid stationary targets in the (a) normal and (b) inclined orientations.

\section{Problem description and simulation methodology}

Consider the normal and oblique impact of a slug comprising granular particles (referred to subsequently as grains) on rigid stationary targets as shown in Fig. 1. The experiments conducted by Goel et al. (2017) indicated that friction between the grains and targets strongly influence the momentum transferred during oblique impacts and that coupled discrete particle/Lagrangian finite element (FE) simulations with spherical grains were unable to capture the measurements with sufficient fidelity. Artificially increasing the rotational inertia of the grains, and thereby reducing the rolling of the grains improved the predictions and suggested that grain shape might have a strong influence on momentum transfer. The objective of this numerical study is to investigate the effect of grain shape on the interactions of high velocity granular media with structures. This is accomplished via coupled discrete particle/Lagrangian finite element simulations performed in the commercial package Abaqus.

Simulations of granular slug impact with rigid stationary targets were conducted in two orientations. In the "normal orientation" (Fig. 1a), the slugs impacted the target at an angle $\alpha=90^{\circ}$ to the face of the target while in the "inclined orientation", the slugs impacted the target at $\alpha=45^{\circ}$ (Fig. 1b). Prior to impact, the granular slugs were cylindrical with a diameter of $12.7 \mathrm{~mm}$ and length $L=50 \mathrm{~mm}$. They comprised a spatially uniform distribution of grains such that the total mass of the slug $m_{\text {slug }}=4.2 \mathrm{~g}$ and the grains occupied a fraction $\bar{\rho} \approx 0.25$ of the slug volume. All the grains were given an initial velocity $v_{0}$ in the negative $z$-direction (Fig. 1) with all other components of the translational and all rotational velocity components set to zero. The rigid stationary targets were $50 \mathrm{~mm}$ wide in the $x$-direction while the length of the target surface facing the slug impact was $50 \mathrm{~mm}$ for normal impact (i.e. a square target of dimensions $50 \mathrm{~mm} \times 50 \mathrm{~mm}$ ) and $100 \mathrm{~mm}$ for inclined impact case, as shown in Fig. 1. The slugs were impacted near the target centre: the targets were sufficiently large such that the transmitted momentum was insensitive to the precise location of the impact. 


\subsection{Geometry of the grains}

Three shapes of grains were used in the calculations presented here: (i) spherical; (ii) rodshaped and (iii) cubic. We emphasize that the aim here is not to simulate a specific soil type but rather illustrate the effect of grain shape on the interaction of high velocity granular media with structures. These grains were all constructed by combining together spherical particles. For clarity of terminology, we shall refer to the spherical particles from which the grains were constructed as sub-particles. The spherical sub-particles are rigid single node spherical elements (PD3D in the Abaqus notation) of density $\rho_{p}$ and diameter $d_{p}$ such that they had a mass $m_{p} \equiv(4 / 3) \pi\left(d_{p} / 2\right)^{3} \rho_{p}$.

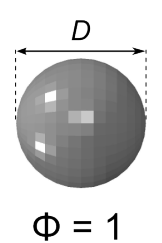

(a)

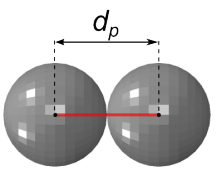

$\Phi=2$

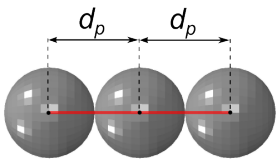

$\Phi=3$

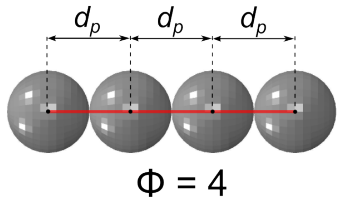

(b)

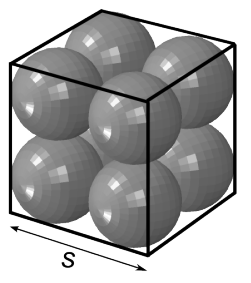

$n=8$

(c)

Figure 2: Sketches of the different grain shapes analysed in this study. (a) Spherical grains of diameter $D$. (b) Rod-shaped grains with aspect ratios $2 \leq \Phi \leq 4$ constructed from spherical sub-particles. (c) Cubic grains comprising $n=8$ spherical sub-particles.

The spherical grains comprised of a single sub-particle of diameter $D$ as shown in Fig. 2a. These spherical grains served as the reference case. The rod-shaped grains comprised a linear array of the sub-particles with centre-to-centre spacing $d_{p}$ as shown in Fig. $2 \mathrm{~b}$. The aspect ratio $\Phi$ of these rod-shaped grains is defined as the ratio of the grain length to the diameter of the circumscribing cylinder. Thus $\Phi=n$, where $n$ is the number of sub-particles that form the grain. In order to perform a study of the effect of the aspect ratio $\Phi$, all grains in the calculations reported here had equal volume. Thus, given the diameter $D$ of the reference spherical grain, the diameter $d_{p}$ of the sub-particles in the rod-shaped grain of aspect ratio $\Phi$ follows as $d_{p}=$ $D / \sqrt[3]{\Phi}$. The cubic grains consisted of a simple cubic packing of the sub-particles such that a cube of side length $s \equiv d_{p} \sqrt[3]{n}$ circumscribes the grain, where $n$ is the number of sub-particles in the grain; see Fig. 2c. Again, the sum of the volume of the sub-particles comprising the cubic grain equals the volume of the reference spherical grain. Thus, the diameter of the sub-particles within cubic grains comprising $n$ sub-particles is $d_{p}=D / \sqrt[3]{n}$.

Two types of connections between the centres of the sub-particles were employed in this study: (i) Rigid connections between the sub-particle centres such that the shape of the grains remained unchanged throughout the impact event. These rigid connections were specified using the multi-point constraint (MPC) functionality in Abaqus.

(ii) The nearest-neighbour centres of the sub-particles were connected to each other using a single Timoshenko beam element (B31 in the Abaqus notation). The inter sub-particle forces were then equal to the sum of the forces due to these beam connections and inter sub-particle contact law as detailed in Section 2.2. These beam elements permitted the grains to deform and fracture (additional details of the beam properties are provided in Section 4). 


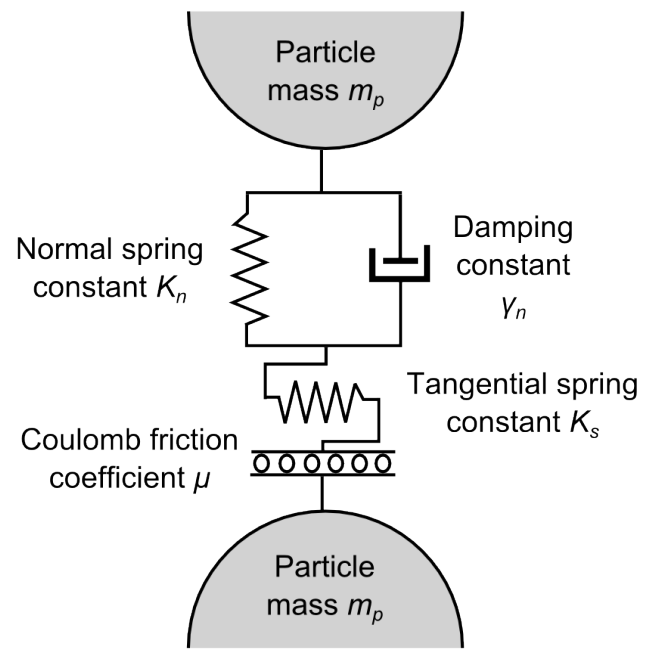

Figure 3: Sketch of the inter sub-particle contact model between spherical sub-particles of diameter $d_{p}$.

\subsection{The particle contact model and coupling to FE simulations}

Since grains we composed of sub-particles in the formulation employed in this study, it is these sub-particles that come into contact with each other as grains collide with each other and/or with the target surface. Thus, it suffices to specify a contact model for sub-particle contact. In line with usual discrete element simulations, the sub-particles were taken to be rigid spheres with contact between the sub-particles and between sub-particles and the target surface modelled using the soft-contact approach (Fig. 3) introduced by Cundall and Strack (1979). The inter sub-particle contact law comprises:

(i) A linear spring with spring constant $K_{n}$ and linear dashpot with damping constant $\gamma_{n}$ connected in parallel, governing the radial contact force-displacement relation (in the direction connecting the sub-particle centres).

(ii) A linear spring of constant $K_{s}$ and Coulomb friction coefficient $\mu$ connected in series, governing the tangential contact relationship.

With $r$ denoting the distance of separation of the sub-particle centres, and the sub-particle interpenetration given by $\delta_{n}=r-d_{p}$, the normal force during active contact $\left(\delta_{n}<0\right)$ is given by

$$
F_{n}=K_{n} \delta_{n}+m_{\mathrm{eff}} \gamma_{n} \dot{\delta}_{n}
$$

where $m_{\text {eff }}$ is the reduced mass of the two contacting bodies. For impacts between subparticles, $m_{\mathrm{eff}}=m_{p} / 2$ while for impacts between a sub-particle and the target, $m_{\mathrm{eff}}=m_{p}$.

The tangential force $F_{S}$ only exists during an active contact, and opposes sliding. It is limited in magnitude to $\left|F_{s}\right|<\mu\left|F_{n}\right|$, where $\mu$ is the friction coefficient and specified as follows. With $\dot{\delta}_{s}$ denoting the tangential displacement rate between the contacting sub-particles, $F_{S}$ is represented by an elastic-plastic relation with stiffness $K_{s}$, i.e.

$$
\dot{F}_{S}= \begin{cases}K_{s} \dot{\delta}_{s} & \text { if }\left|F_{s}\right|<\mu\left|F_{n}\right| \text { or } F_{s} \dot{\delta}_{s}<0 \\ 0 & \text { otherwise. }\end{cases}
$$


The critical difference for interactions between sub-particles and between a sub-particle and the target is the coefficient of friction $\mu$ : we use $\mu_{p}$ and $\mu_{T}$ to denote the values of $\mu$ for inter sub-particle and sub-particle/target interactions, respectively. The value of damping constant $\gamma_{n}$ dictates the loss of sub-particle kinetic energy during normal collision which is conveniently parameterised in terms of the coefficient of restitution $e$. This co-efficient is obtained by solving the Netwon's equations for a pair of particles with the force between the particles set by (2.1). Cundall and Strack (1979) give the coefficient of restitution $e$ as

$$
e=\exp \left[-\pi\left(\frac{8 K_{n}}{\gamma_{n}^{2} m_{p}}-1\right)^{-1 / 2}\right]
$$

while the collision time $t_{e}$ for individual binary collisions follows as

$$
t_{e}=-2 \frac{\ln (e)}{\gamma_{n}} \text {. }
$$

Thus, in the limit of plastic collisions $\left(e \rightarrow 0\right.$ ), the contact time $t_{e} \rightarrow \infty$. The forces (normal and tangential) on each sub-particle are calculated at each time step and the Newton's equations for both the translational and rotational motions of the sub-particles are integrated using an explicit time-integration scheme provided in Abaqus. The time-step for integration was taken to be less than $t_{e} / 10$ in order to ensure accurate integration of the equations of motion.

The rigid targets were modelled as analytical rigid surfaces and all six degrees of freedom (3 rotational and 3 translational) constrained to prevent all rigid body motions. The coupling between the discrete grains and rigid surfaces was performed as follows. At a given time $t$, a proportion of the grains are in contact with the target surface. Consider one such grain in contact with the target. One or more of the sub-particles of that grain are in contact with the target with an interpenetration $\delta_{n}$ defined as $\delta_{n}=r-d_{p} / 2$, where $r$ is the distance between the sub-particle centre and contact point on the target (note the difference in the definition of $\delta_{n}$ for inter sub-particle and sub-particle/target interactions). Denote $\dot{\delta}_{n}$ to be the relative approach velocity of the sub-particle and the point of contact on the target surface, and likewise $\dot{\delta}_{S}$ is the relative tangential velocity. The normal and tangential contact forces are then calculated using Eqs. (2.1) and (2.2). The targets were modelled using a Lagrangian finite element (FE) framework and these forces were then added as nodal forces in the finite element analysis of the target to complete the coupling between the discrete and finite element calculations.

The primary goal of this study was to determine the momentum transmitted from the impacting slug into the target. Denote the reaction forces in the $x$-, $y$ - and $z$-directions on the target as $F_{x}, F_{y}$ and $F_{z}$, respectively. Then, the momentum $i_{T}^{z}$ transmitted into the target in the $z$ direction after a time $t$ (where $t=0$ corresponds to the instant that the slug first comes into contact with the target) is given by

$$
i_{T}^{Z}(t)=\int_{0}^{t} F_{z} d t^{\prime}
$$

and $I_{T}^{Z} \equiv i_{T}^{Z}$ (in the limit $t \rightarrow \infty$ ) is the total momentum transmitted into the target in the $z$ direction. Analogous expressions exist for momentum components in the other two orthogonal directions (viz. $I_{T}^{y}$ and $i_{T}^{y}$ as well as $I_{T}^{x}$ and $i_{T}^{x}$ ). For both normal and oblique impacts analysed here, symmetry dictates that $i_{T}^{x}=0$ and so we focus on the momentum in the $z$ - and $y$ directions and the resultant transmitted momentum given by 


$$
I_{T}=\sqrt{\left(I_{T}^{z}\right)^{2}+\left(I_{T}^{y}\right)^{2}}
$$

All results are presented with the transmitted momenta normalised by the initial momentum $I_{0} \equiv m_{\text {slug }} v_{0}$ of the slug in the $z$-direction.

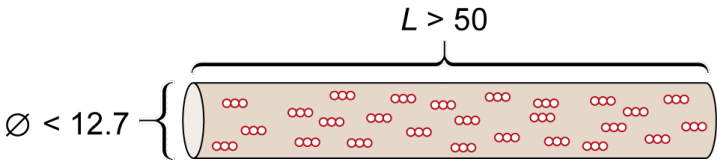

(a)

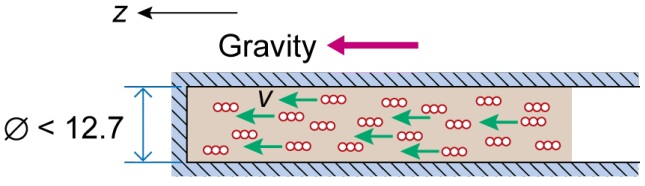

(b)

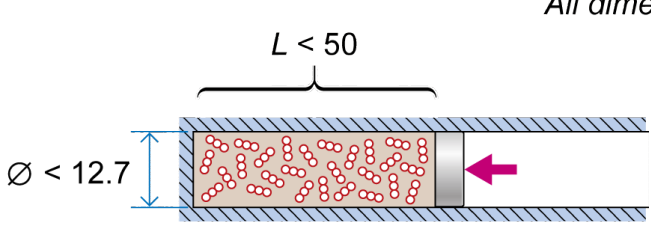

(c)

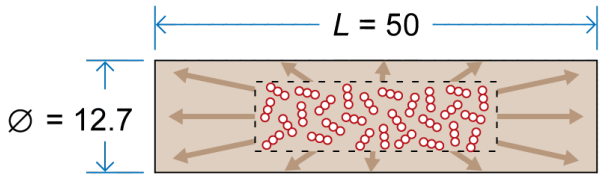

(d)

Figure 4: Sketches illustrating the construction of the cylindrical granular slug. (a) Step (i): randomly located but aligned grains. (b) step (ii): settling of the grains under the influence of gravity. (c) step (iii): compaction of the grains in the cylindrical cavity. (d) step (iv): affine expansion to achieve the required size of the slug.

\subsection{Construction of granular slugs}

Prior to impact, the cylindrical slugs have a spatially uniform distribution of identical grains that occupy a volume fraction $\bar{\rho}$. While all grains have the same initial velocity, we desire that the grains are located and oriented randomly within the slug. This was achieved via a four-step process shown schematically in Fig. 4 so as to obtain cylindrical slugs with a diameter 12.7 mm and length $L=50 \mathrm{~mm}$.

(i) The grains were first randomly located within a cylinder of diameter $<12.7 \mathrm{~mm}$ and length $L>50 \mathrm{~mm}$. In this step, while the location of the grains was random, all grains had the same orientation, i.e. the rod-shaped grains were oriented along the axis of the cylinder (Fig. 4a) while the cubic grains had two faces parallel to the flat faces of the cylinder. This process typically resulted in a slug with a grain volume fraction $\ll \bar{\rho}$.

(ii) All grains were then given a velocity $v=10 \mathrm{~m} \mathrm{~s}^{-1}$ in the $z$-direction and the end of the cylinder towards which the grains were travelling was capped by a rigid face (Fig. 4b). The grains were then allowed to collide with each other, dissipate their kinetic energy via plastic collisions (using contact properties specified in Section 2.4) and settle within the cylinder under the influence of gravity that was acting in the positive $z$ direction.

(iii) The grains were then further compacted within the cylinder by the action of a piston as shown in Fig. 4c. The collisions and re-arrangements of the grains in steps (ii) and (iii) resulted in a dense random packing of the grains within a cylindrical volume of length $<50 \mathrm{~mm}$.

(iv) At the end of step (iii), the dense randomly packed grains were within a cylindrical volume with both diameter and length less than the desired values. We then applied a hydrostatic affine transformation (Fig. 4d), i.e. the centroid of each grain was displaced by $u_{i}=(H / 3) \delta_{i j} x_{j}$, where $H$ and $\delta_{i j}$ are the hydrostatic strain and Kronecker delta, respectively while $x_{j}$ is the Cartesian location of the grain centroid. The hydrostatic 
strain $H$ was chosen such that the slug attained the desired diameter and length after application of the transformation. We emphasize that iterations were required wherein the initial diameter and length of the slug in step (i) were adjusted such that the final slug had the required overall dimensions.

The slugs thus comprised identical particles with all grains having equal velocity $v_{0}$ prior to impact. Such an idealisation, while unrealistic from a practical standpoint, was employed to enable a detailed investigation of the effect of particle shape and fracture without the additional complications of variabilities in field experiments. It is worth emphasizing that while a relatively small slug size is used in the calculations reported here, the number of grains in the slug is sufficiently large so as to attain a "continuum limit"; see Pingle et al., (2012) for a discussion on slug size effects.

\subsection{Material parameters}

All calculations presented here used the following reference properties for the grains and subparticles. The granular slugs had a volume fraction $\bar{\rho}=0.25$ of grains and the reference spherical grain had a diameter $D=600 \mu \mathrm{m}$, i.e. the volume of the individual grains was equal to that of a sphere of diameter $600 \mu \mathrm{m}$. The sub-particles were assumed to be made from a solid of density $\rho_{p}=2700 \mathrm{~kg} \mathrm{~m}^{-3}$ (approximately equal to that of the quartz phase of silica) and the sub-particle contact model was defined by four parameters $K_{n}, e, K_{s}$ and $\mu_{p}$. Liu et al. (2013) and Goel et al. (2017) demonstrated that these parameters have a negligible effect on the interaction of the granular slug with the targets and thus following these studies we chose them to be $K_{n}=0.69 \mathrm{MN} \mathrm{m}^{-1}, K_{s} / K_{n}=2 / 7$ (Bathurst and Rothenburg, 1988; Silbert et al., 2001), $e=0.7$ and $\mu_{p}=0.6$. The friction coefficient $\mu_{T}$ between the particles and the target surface depends on the target surface and was varied over the range $0.0 \leq \mu_{T} \leq 2.0$ in the calculations presented here. The effect of gravity was neglected in all the impact simulations that are discussed subsequently.

The calculations presented in Section 3 use rigid non-deformable grains and the properties listed above completely specify the required material properties. The calculations in Section 4 allow the grains to deform and fracture by employing sub-particles connected together by deformable beams. The material properties for these beams are presented along with the calculations in Section 4. As a rough estimate, each calculation with non-deformable grains reported subsequently required around 1 to 10 core-hours of computational time while the calculations with grains that deform and fracture were computationally more expensive and requiring between 20 to 60 core-hours.

\section{Effect of particle shape}

We proceed to present numerical results for the effect of grain shape on the interaction of high velocity granular slugs with the targets in both the normal and inclined orientations. Since the objective is to investigate only the effect of grain shape, all the simulations were restricted to grains comprising either a single spherical particle or grains constructed by joining together sub-particles via rigid connections. A number of studies (Goel et al., 2017; Liu et al., 2013; Pingle et al., 2012) have shown that for slugs comprising spherical grains, the transmitted momentum normalised by the initial slug momentum $I_{0}$, is independent of the slug velocity $v_{0}$. Here, we confirmed via spot calculations that this result extrapolates to non-spherical rigid grains that do not fracture. Thus, all numerical simulations presented in this section were conducted with $v_{0}=100 \mathrm{~m} \mathrm{~s}^{-1}$ and the calculated transmitted momentum is presented in 
normalised form, i.e. the momentum transmitted into the structure is normalised by the initial momentum $I_{0}$ of the slug.

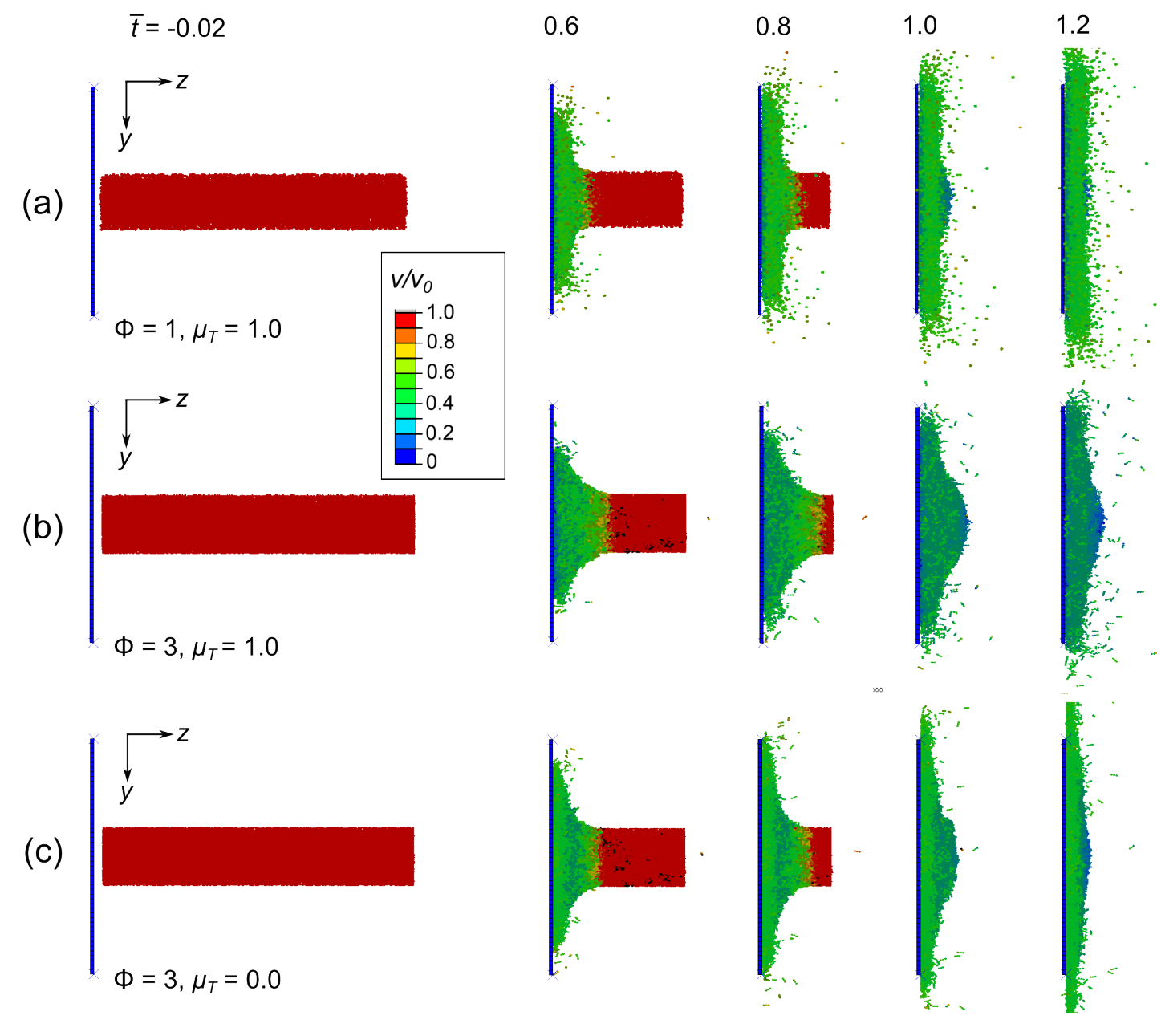

Figure 5: Predictions of the flow of granular slugs impacting normally oriented targets. Results are shown at four selected values of the normalised time $\bar{t}$ where $\bar{t}=0$ is the instant the slug begins impact with the target. (a) Spherical grains with $\mu_{T}=1.0$, (b) $\Phi=3$ rod-shaped grains with $\mu_{T}=1.0$ and (c) $\Phi=3$ rod-shaped grains with $\mu_{T}=0$. In all the cases, the grains are coloured by the magnitudes of their velocity $v$ normalised by the initial slug velocity $v_{0}$.

\subsection{Normal impact on targets}

Snapshots showing the flow of the granular slug impacting the normally oriented rigid stationary target are shown in Fig. 5 for selected values of the dimensionless time $\bar{t} \equiv t v_{0} / L$, where time $t=0$ is defined as the instant the slug begins impact with the target. The grains are coloured by the magnitude of the grain velocity $v$ normalised by $v_{0}$. First, consider the reference case of spherical grains $(\Phi=1)$ with a friction coefficient $\mu_{T}=1.0$ between the grains and target surface (Fig. 5a). The flow is reminiscent of a water jet impinging normally on a rigid stationary surface with the grains flowing along the target surface in the $y$-direction. The zone over which the grains change their direction of motion from the positive $z$-direction to the $y$-direction is negligibly small with no stagnation zone visible in Fig. 5a. Next, consider the case of impact by a slug with rod-shaped grains with an aspect ratio $\Phi=3$. The predictions of the flow patterns with $\mu_{T}=1.0$ and no friction $\left(\mu_{T}=0\right)$ between the grains and target 
surface are included in Figs. 5b and 5c, respectively. The velocity with which the grains spread along the $y$-direction is lower for the case with $\mu_{T}=1.0$. This is seen in not only the magnitudes of the velocities of the grains (compare Fig. 5b with Figs. 5a and 5c) but also evident in the lower level of spreading seen at $\bar{t}=0.8$ in Fig. 5b compared to both Figs. 5a and $5 \mathrm{c}$. This can be understood by recalling that rotation of the rod-shaped grains requires work to be done against the hydrodynamic pressure exerted by the incoming high velocity grains. This impedes the rotation rate of these grains and the grains instead increasingly slide rather than roll along the target surface in the $y$-direction. A high friction between the target and the grains then resists this sliding motion and results in the reduction in the velocity with which the grains spread along the target. In contrast, the hydrodynamic pressure exerted by the incoming grains is less effective in impeding the rotation of spherical grains, and thus friction has only a small influence on the flow of these grains along the target surface.

We now proceed to quantify the transmitted momentum. The symmetry of the normal impact implies that $I_{T}^{y} \approx I_{T}^{x} \approx 0$ (the approximate signs are used to emphasize that the stochastic nature of the granular slug means that the impact is not perfectly symmetrical in the numerical calculations), and thus the total transmitted momentum $I_{T} \approx I_{T}^{Z}$. Calculations of the normalised transmitted momentum $I_{T} / I_{0}$ are shown in Fig. 6a as a function of grain aspect ratio $\Phi$ for selected values of $\mu_{T}$. Consistent with conservation of momentum and negligible rebound of the grains (Fig. 5), $I_{T} / I_{0} \approx 1$ in all cases, i.e. the changes in the flow patterns with grain shape seen in Fig. 5 do not affect the transmitted momentum for normal impacts. However, as seen in Fig. 5, friction between the target surface and grains, and the shape of the grains affects the flow pattern. We thus anticipate these parameters to influence the forces transmitted into the target in the $y$-direction. To investigate this, we define a measure $J_{T}^{y}$ of the momentum transmitted into the target in the $y$-direction as follows. We divide the target into two halves about the axis of symmetry as shown in the inset of Fig. $6 \mathrm{~b}$ and calculate the total force exerted by the grains on each half of the target in the $y$-direction. These forces are labelled $f_{b}^{y}$ and $f_{u}^{y}$ where the subscripts " $b$ " and " $u$ " denote the bottom and upper halves of the target, respectively. Then $J_{T}^{y}$ is calculated as

$$
J_{T}^{y} \equiv \lim _{t \rightarrow \infty} \int_{0}^{t}\left(\left|f_{b}^{y}\left(t^{\prime}\right)\right|+\left|f_{u}^{y}\left(t^{\prime}\right)\right|\right) d t^{\prime}
$$
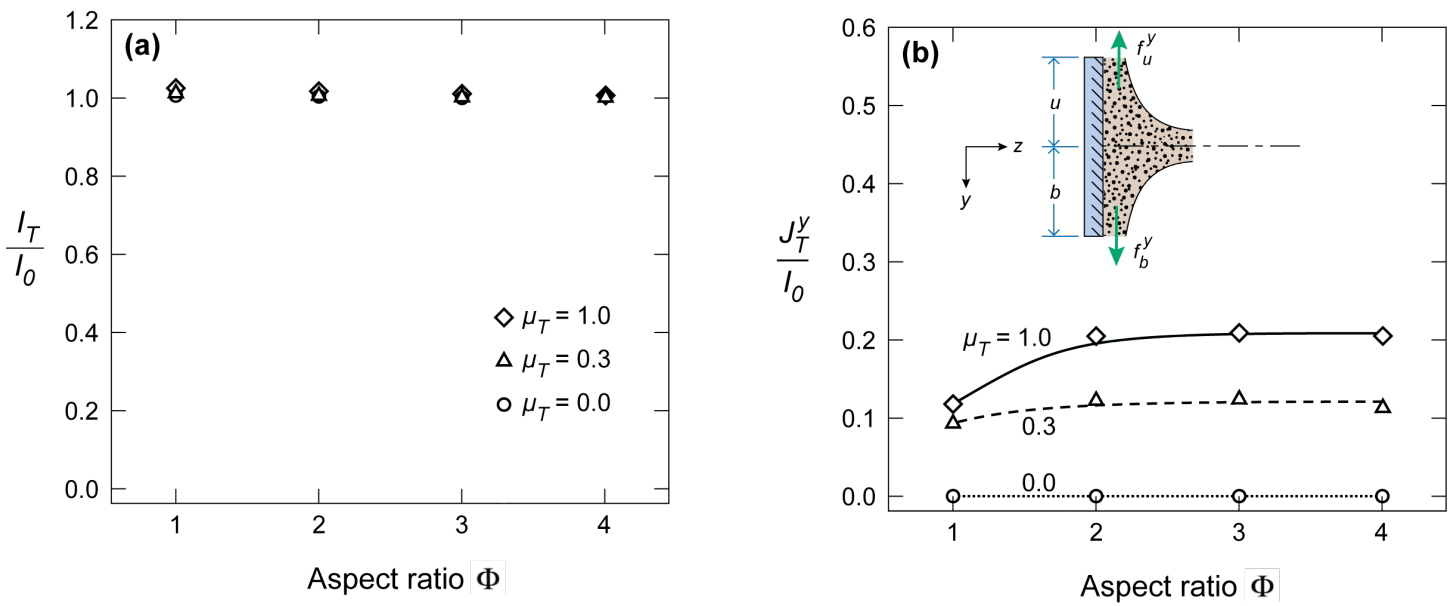

Figure 6: Predictions of the (a) normalised total transmitted momentum $I_{T} / I_{0}$ and (b) the measure $J_{T}^{y} / I_{0}$ of the normalised momentum transmitted as a function of the aspect ratio $\Phi$ of the grains. These results are shown for three selected values of $\mu_{T}$ for the case of the normally oriented target. The inset 
in (b) shows the target divided into the upper and bottom halves for the purposes of the calculation of $J_{T}^{y}$.

This definition of $J_{T}^{y}$ negates the symmetry of the problem by neglecting the sign of the momentum transferred into the bottom and upper halves of the target and thus unlike $I_{T}^{y}$ does not vanish. Predictions of $J_{T}^{y} / I_{0}$ are included in Fig. $6 \mathrm{~b}$ for the cases analysed in Fig. 6a. When $\mu_{T}=0$, no momentum can be transferred by the grains into the target in the $y$-direction, resulting in $J_{T}^{y}=0$ for all aspect ratios $\Phi$. However, $J_{T}^{y}$ increases with increasing $\mu_{T}$ and with grain aspect ratio $\Phi$. This increase is mainly over the range $1 \leq \Phi \leq 2$ with increasing the aspect ratio beyond $\Phi=2$ resulting in no further increases in $J_{T}^{y}$. We rationalise this by noting that the momentum transferred from the grains to the target in the $y$-direction is a maximum when the grains undergo pure sliding along the target surface while this transfer of momentum vanishes if the grains undergo pure rolling. The results in Fig. $6 \mathrm{~b}$ suggest that while the spherical grains move along the target surface by a combination of rolling and sliding, rolling of the grains is eliminated for $\Phi \geq 2$. Thus, increases in $\Phi$ beyond $\Phi=2$ has no influence on $J_{T}^{y}$.

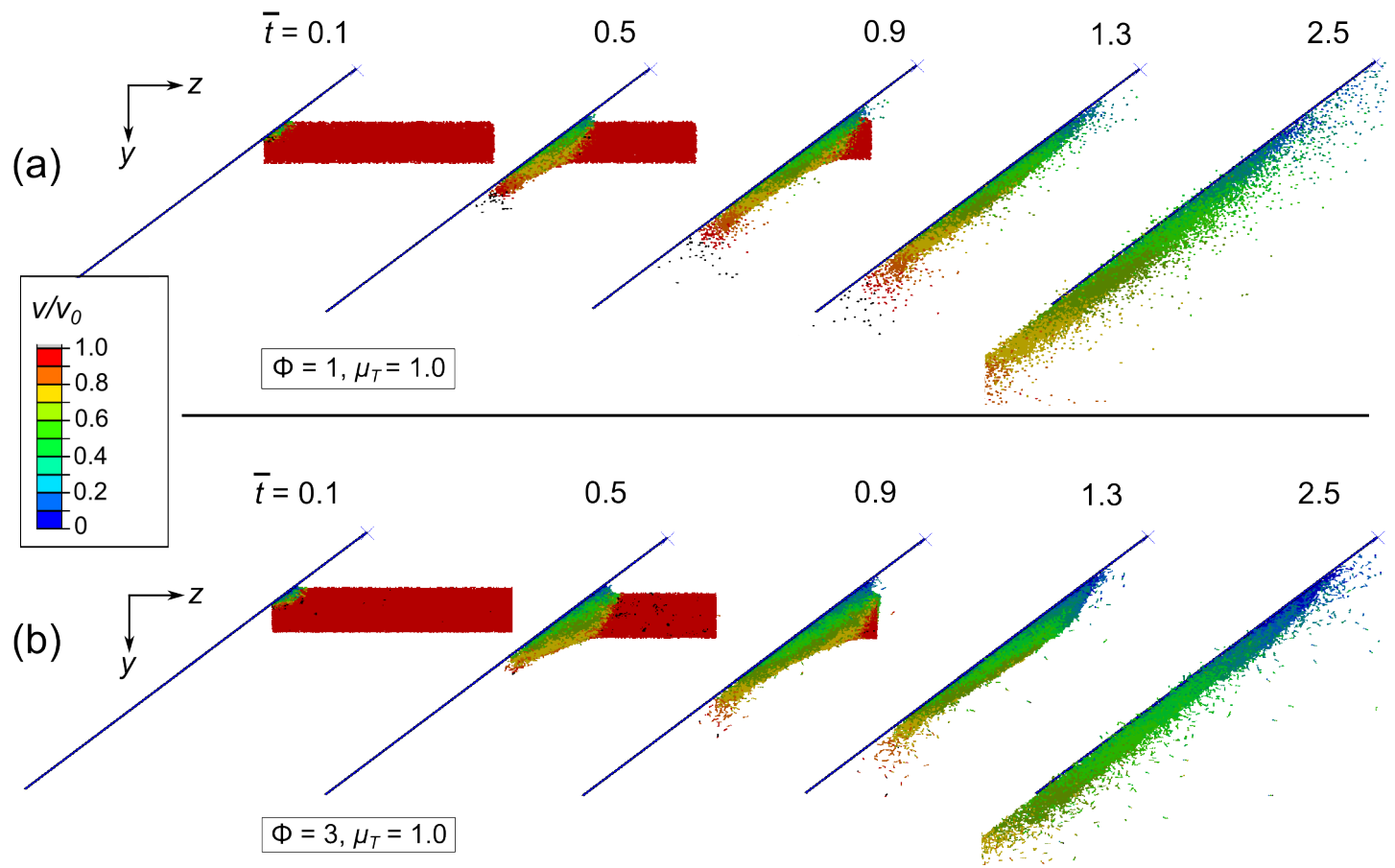

Figure 7: Predictions of the flow of the granular slug impacting the inclined $\left(\alpha=45^{\circ}\right)$ target at selected values of the normalised time $\bar{t}$ where $\bar{t}=0$ is the instant the slug begins impact with the target. Results are shown for slugs comprising (a) spherical grains and (b) $\Phi=3$ rod-shaped grains with $\mu_{T}=1.0$ in both cases. In all cases, the grains are coloured by the magnitudes of their velocity $v$ normalised by the initial slug velocity $v_{0}$.

\subsection{Impact with inclined targets}

Before proceeding to present numerical results for granular slugs impacting inclined targets, it is instructive to discuss some of the basic physics of momentum transfer from granular slugs into inclined targets. Let $f_{n}$ and $f_{s}$ denote the resultant normal and tangential forces exerted by the grains on the target (Fig. 1b). Momentum balance then gives 


$$
i_{T}^{z}(t)=\int_{0}^{t}\left(f_{n} \sin \alpha+f_{s} \cos \alpha\right) d t^{\prime}
$$

and

$$
i_{T}^{y}(t)=\int_{0}^{t}\left(f_{n} \cos \alpha-f_{s} \sin \alpha\right) d t^{\prime}
$$

with $I_{T}^{z} \equiv i_{T}^{z}(t \rightarrow \infty)$ and $I_{T}^{y} \equiv i_{T}^{y}(t \rightarrow \infty)$. It is evident that the tangential force (resulting from the friction between the grains and the target) has opposite effects on $i_{T}^{z}$ and $i_{T}^{y}$ and for $\alpha=45^{\circ}$ we anticipate that $i_{T}^{z}>i_{T}^{y}$. The consequences of Eqs. (3.2) and (3.3) in the limiting case when $t \rightarrow \infty$ are also worth further elaboration as it enables better interpretation of numerical predictions reported subsequently. For the case when there are frictional interactions between the grains and the target surface (i.e. $\mu_{T}>0$ ), we cannot directly relate $I_{T}^{Z}$ and $I_{T}^{y}$ to $I_{0}$ without detailed information on $f_{s}$. However, in the absence of friction $\left(\mu_{T}=0\right), f_{s}=0$ and explicit relations for the transmitted momentum follow as

$$
I_{T}^{z}=I_{0} \sin ^{2} \alpha \quad \text { and } \quad I_{T}^{y}=I_{0} \sin \alpha \cos \alpha .
$$

For the $\alpha=45^{\circ}$ case these reduce to $I_{T}^{Z} / I_{0}=I_{T}^{y} / I_{0}=0.5$ with $I_{T} / I_{0}=1 / \sqrt{2}$. These values will subsequently be used as a reference to aid the quantification of the effect of friction.

Simulations of the flow of granular slugs with spherical or rod-shaped grains $(\Phi=3)$ are shown in Figs. $7 \mathrm{a}$ and $7 \mathrm{~b}$, respectively for the case $\mu_{T}=1.0$. The grains are coloured by the magnitude of the grain velocity $v$ normalised by $v_{0}$. The grains flow along the inclined surface in both directions although the primary flow is in the positive $y$-direction. While differences between the flow patterns in Figs. $7 \mathrm{a}$ and $7 \mathrm{~b}$ are not immediately evident, a careful comparison of the flow patterns in Figs. $7 \mathrm{a}$ and $7 \mathrm{~b}$ at $\bar{t}=0.9$ indicates that the granular flow is slower for the rod-shaped grains. This is again associated with inhibition of rod-shaped grain rolling which results in higher levels of sliding friction between the grains and the target surface.
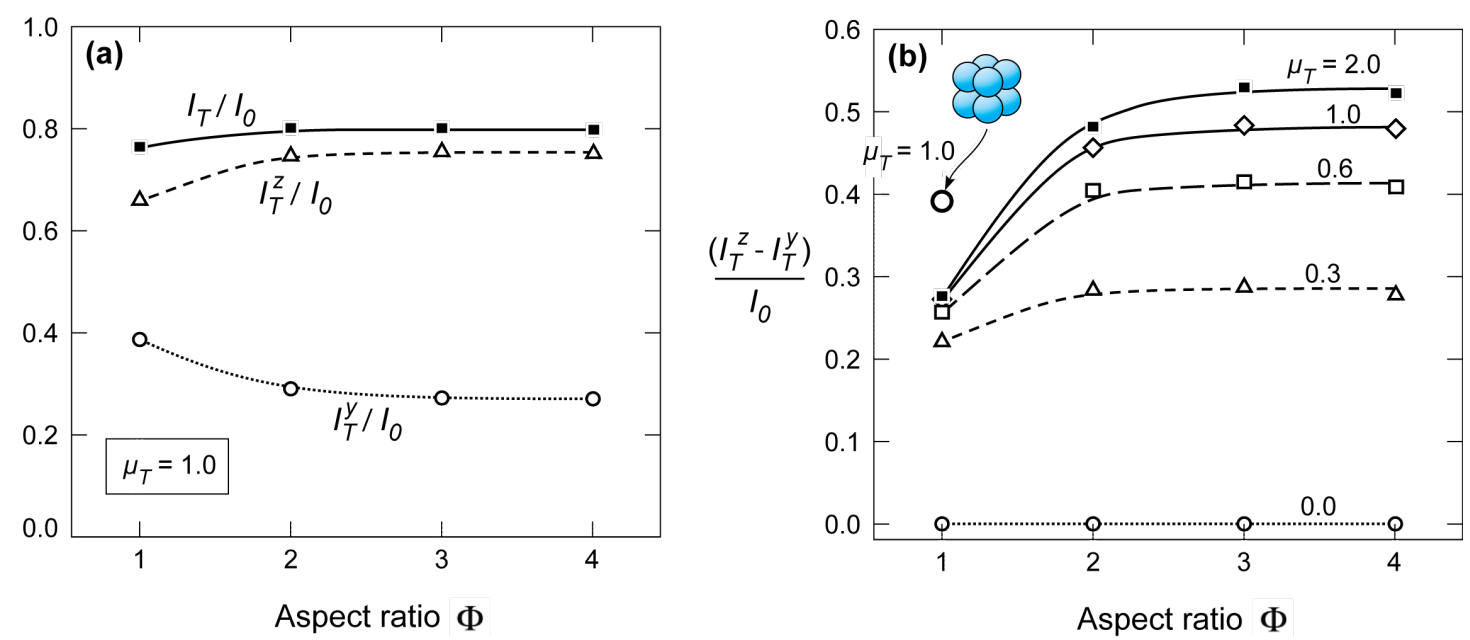

Figure 8: Predictions of the normalised transmitted momenta (a) $I_{T} / I_{0}, I_{T}^{Z} / I_{0}$ and $I_{T}^{y} / I_{0}$ and (b) $\left(I_{T}^{Z}-\right.$ $\left.I_{T}^{y}\right) / I_{0}$ for slugs impacting the inclined $\left(\alpha=45^{\circ}\right)$ target as a function of the grain aspect ratio $\Phi$. In (a) results are shown for $\mu_{T}=1.0$ while in (b) we include predictions for selected values of $\mu_{T}$. The prediction of $\left(I_{T}^{Z}-I_{T}^{y}\right) / I_{0}$ for a slug with cubic grains comprising 8 sub-particles and $\mu_{T}=1.0$ is also included in (b). 
Unlike for normal impact, the transmitted momentum is also strongly dependent on grain shape for inclined impacts. Predictions of the normalised transmitted momenta $I_{T} / I_{0}, I_{T}^{Z} / I_{0}$ and $I_{T}^{y} / I_{0}$ are shown in Fig. 8a as a function of $\Phi$ for the case with $\mu_{T}=1.0$. As anticipated from Eqs. (3.2) and (3.3), $I_{T}^{z} / I_{0}>I_{T}^{y} / I_{0}$ for all $\Phi$. Moreover, $I_{T}^{Z} / I_{0}$ increases but $I_{T}^{y} / I_{0}$ decreases with increasing $\Phi$. This can be rationalised as follows. With increasing $\Phi$, the grains increasingly slide rather than roll along the target surface thereby increasing frictional interactions with the target. Since, the primary flow of the grains is in the positive $y$-direction, increasing frictional interactions reduces $I_{T}^{y}$ while increasing $I_{T}^{Z}$. The outcome of these changes in $I_{T}^{y}$ and $I_{T}^{Z}$ is that the total transmitted momentum $I_{T} / I_{0}$ increases with $\Phi$ (albeit to a small extent). Recall that in the absence of frictional interactions between the grains and the target surface, $I_{T} / I_{0}=1 / \sqrt{2}=0.71$. Here, we predict for $\mu_{T}=1.0$ that the total transmitted momentum increases from $I_{T} / I_{0}=0.76$ for spherical grains to 0.80 for rod-shaped grains with $\Phi=4$. Thus, frictional interactions increase the transmitted momentum into inclined targets for both spherical and rod-shaped grains. These frictional interactions can be parameterised by $\left(I_{T}^{Z}-I_{T}^{y}\right)$, which from Eq. (3.4), vanishes in the absence of friction between the grains and a target surface inclined at $\alpha=45^{\circ}$. Predictions of $\left(I_{T}^{Z}-I_{T}^{y}\right) / I_{0}$ are presented in Fig. $8 \mathrm{~b}$ as a function of $\Phi$ and selected values of $\mu_{T}$. Clearly, $\left(I_{T}^{Z}-I_{T}^{y}\right)$ not only increases with $\mu_{T}$ but also with $\Phi$ (for non-zero values of $\mu_{T}$ ) due to the increasing levels of frictional interaction. However, consistent with the predictions for the normal impact case, the dependence on $\Phi$ is minimal for $\Phi>2$ : these high aspect ratio grains undergo pure sliding along the target surface and thus further increases in $\Phi$ have a negligible influence on the frictional interactions between the grains and the target surface.

All calculations reported up to this point were restricted to spherical and rod-shaped grains whose shape is parameterised by the aspect ratio $\Phi$. However, it is worth emphasising that the frictional interactions resulting from the competition between the rolling and sliding of the grains along the target surface depends on the precise shape of the grains with $\Phi$ insufficient to fully characterise these interactions. To illustrate this, we consider a slug comprising cubic grains constructed with 8 spherical sub-particles (i.e. cubic grains with $n=8$ ). The aspect ratio of this grain is defined to be $\Phi=1^{1}$, i.e. equal to that of a spherical grain. The prediction of $\left(I_{T}^{z}-I_{T}^{y}\right) / I_{0}$ for a slug comprising cubic grains with $\mu_{T}=1.0$ included in Fig. $8 \mathrm{~b}$ shows that $\left(I_{T}^{Z}-I_{T}^{y}\right) / I_{0}$ is $42 \%$ above that of the case with spherical grains. This is because the rolling resistance of cubic grains is significantly higher than that of spherical grains resulting in cubic grains having a higher tendency to slide along the target surface. The sliding of the grains increases the frictional interactions between the grains and the target and consequently increases $\left(I_{T}^{Z}-I_{T}^{y}\right)$. This clearly illustrates that transmitted momenta depend on the precise shape of the grains with a simple parameter like aspect ratio insufficient to characterise the grains for the purposes of determining the transmitted momentum.

\section{Effect of particle fragmentation}

The calculations reported so far assume that the grains are rigid and do not fracture upon impact. However, it is well established that high velocity granular impact results in fragmentation of the grains (Parab et al., 2014; Potapov and Campbell, 1997; Samimi et al., 2004; Subero and Ghadiri, 2001) although the effect, if any, on the momentum transfer is not

\footnotetext{
${ }^{1}$ In general, the aspect ratio of a grain is defined as the ratio of the lengths of the semi-major and smallest semi-minor axes of the best-fit ellipsoid to the grain.
} 
understood. We proceed to investigate the effect of grain fragmentation on the momentum transfer for slugs impacting the $\alpha=45^{\circ}$ inclined targets with a target surface/grain friction coefficient $\mu_{T}=1.0$. We shall show that effect of grain fragmentation on the momentum transfer emanates from a change in the shape of the grains. Since grain shape does not affect $I_{T} / I_{0}$ for normal impact (Section 3.1), attention here is restricted to the inclined impact case.

\subsection{Numerical scheme for grain fragmentation}

The breaking/fragmentation of the grains was implemented by connecting the sub-particles to each other via beam elements rather than rigid connections as described in Section 2.1. These beams were not designed to model a specific physical fracture process but rather serve as a numerical tool to permit the splitting of grains during the impact event. Thus, the properties of the beams were chosen to permit a parametric study rather than related to fracture properties a particular granular medium (e.g. silica sand). Moreover, rather than complete shattering of grains, as observed in some impact scenarios, the numerical model here breaks a single rodshaped grain into at most 3 sub-particles and a cubic grain into two flake-like grains, each comprising 4 sub-particles. Thus, the results here should be viewed as qualitatively indicative of the effect of grain fragmentation rather than for quantitative predictive ability.

The nearest-neighbour centres of the sub-particles were connected to each other via a single ${ }^{2}$ Timoshenko beam element (B31 in the Abaqus notation) with a circular cross-section of diameter $d_{b}$. Built-in connections were specified at the ends of the beam at the sub-particle centres. The beams were assumed to be made from an elastic perfectly plastic material with Young's modulus $E_{b}$, Poisson ratio $v_{b}$, yield strength $Y_{b}$ and plastic failure strain $\varepsilon_{f}$ while the density of the beams was chosen such that the mass of all the beams in a grain was less than $1 \%$ of the total grain mass. The Poisson's ratio was set to $v_{b}=0.17$ and yield strain $\varepsilon_{Y} \equiv$ $Y_{b} / E_{b}=0.1 \%$ in all the calculations reported here. Since the beams connect the sub-particle centres, the beam length was fixed at $d_{p}$ and thus the beams are fully specified through the beam aspect ratio $d_{p} / d_{b}, E_{b}$ and $\varepsilon_{f}$. We report parametric calculations for the effect of $\varepsilon_{f}$ with $d_{p} / d_{b}$ and $E_{b}$ selected such that in the absence of plasticity and failure, we recover the limiting case of rigid grains. Calibration calculations discussed in the Appendix show that the choice $d_{p} / d_{b}=1$ and $\bar{K}_{b}=10$ is adequate to recover this rigid limit where

$$
\bar{K}_{b} \equiv \frac{\pi E_{b} d_{b}}{4 K_{n}}\left(\frac{d_{b}}{d_{p}}\right)
$$

is the ratio of the axial stiffness of the beam to the normal contact stiffness between the subparticles. All calculations reported in this section use these properties for the beam connectors between the sub-particles. Moreover, since the level of fragmentation depends on the slug impact velocity $v_{0}$, we shall show that unlike in the case of rigid grains, the transmitted momentum now depends on $v_{0}$. Thus, here we present predictions for a range of normalised impact velocities $\bar{v}_{0} \equiv v_{0} / \sqrt{Y_{b} / \rho_{p}}$, where $\rho_{p}$ is the density of the sub-particle material.

\subsection{Summary of numerical results}

It was shown in Section 3.2 that increasing the grain aspect ratio increases momentum transfer from a slug into inclined targets due to an increase in the sliding of the grains on the target surface. We therefore anticipate that the primary effect of grain fragmentation is related to the

\footnotetext{
${ }^{2}$ By using a single beam element, we do not capture stress concentrations that develop between the sub-particles. Thus, the calculations can only be used to qualitatively understand the influence of grain fracture with the fracture properties of the beams not related to realistic material parameters.
} 
change in the grain shape and with this in mind we consider the following two cases: high aspect ratio grains fragmenting to low aspect ratio grains and vice versa.

\subsubsection{Fragmentation from high to low aspect ratio grains}

To investigate the effect of high aspect ratio grains fragmenting to lower aspect ratio grains we restrict attention to the $\Phi=3$ rod-shaped grains with all the rigid connections between the subparticles replaced by the beams described above. Predictions of the total transmitted momentum $I_{T} / I_{0}$ are included in Fig. 9a as a function of the failure strain $\varepsilon_{f}$ of the beam connectors. Results are included for three choices of the normalised slug impact velocity $\bar{v}_{0}$. The $I_{T} / I_{0}$ versus $\varepsilon_{f}$ relation is reasonably independent of $\varepsilon_{f}$ for $\varepsilon_{f}<10 \%$ and about $10 \%$ lower than that for the rigid grains (this limit is indicated in Fig. 9a). At higher failure strains, $I_{T} / I_{0}$ for the two lower impact velocities rises and attains the value for the rigid grains. However, for the highest impact velocity of $\bar{v}_{0}=0.226, I_{T} / I_{0}$ rises only by about $1.5 \%$ over the entire range of $\varepsilon_{f}$ investigated here.
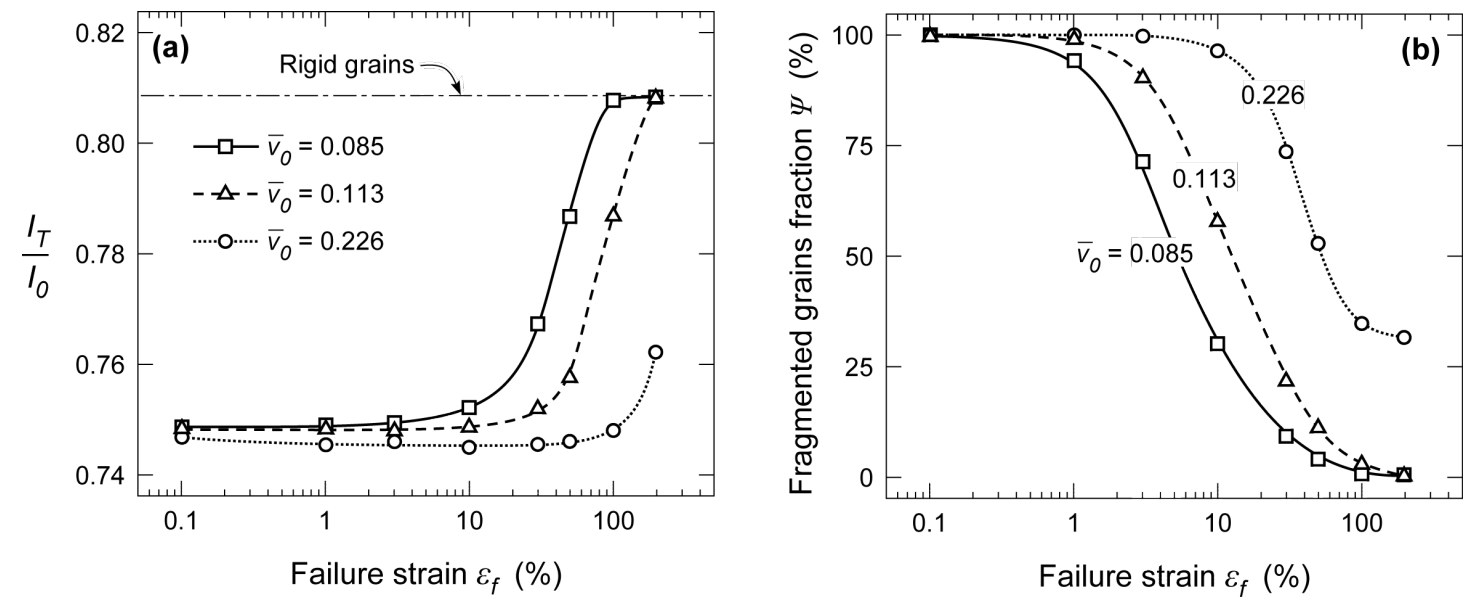

Figure 9: Predictions of (a) the normalised transmitted momentum $I_{T} / I_{0}$ and (b) fraction $\psi$ of the fragmented grains as a function of the failure strain $\varepsilon_{f}$ for a slug comprising $\Phi=3$ rod-shaped grains impacting inclined $\left(\alpha=45^{\circ}\right)$ targets. The predictions are shown for $\mu_{T}=1.0$ and three selected values of the normalised impact velocity $\bar{v}_{0}$.

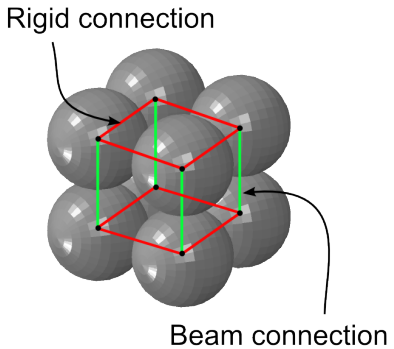

(a)

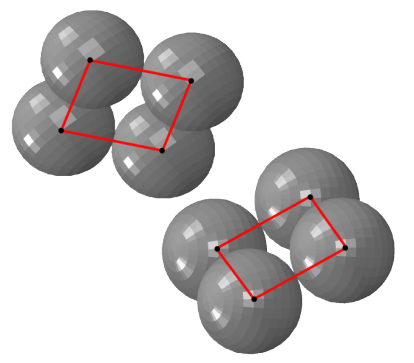

(b)

Figure 10: Sketches of the cubic grain comprising 8 sub-particles used to investigate the effect of grain fracture. (a) The cubic grain with 12 connections between sub-particles. The 4 beam connectors are indicated in green while the remainder of the connectors are rigid and marked in red. (b) The flake-like grains resulting from the fracture of the 4 beam connectors.

To understand these dependencies, we include in Fig. 9b the corresponding predictions of the fraction $\psi$ of fragmented grains after the impact event. Here, we define a grain to be fragmented if at least one of the beam connections present in the original $\Phi=3$ rod-shaped 
grain had failed. The $\psi$ versus $\varepsilon_{f}$ relation has a sigmoidal shape such that $\psi \approx 100 \%$ at low $\varepsilon_{f}$, i.e. at low failure strains all the grains in the impacting slug had fractured. This results in the grains converting from $\Phi=3$ rod-shaped grains to more spherical grains which have a higher tendency to roll rather than slide along the target surface. As seen in Section 3.2, the momentum transfer is lower for more spherical grains and thus an increase in grain facture reduces momentum transfer from slugs with high aspect ratio grains. With increasing $\varepsilon_{f}$ the fraction of grains that fracture decreases with nearly no grain fracturing for the lower two impact velocities when $\varepsilon_{f}$ exceeds $100 \%$. Thus, the transmitted momentum increases to attain the value for the rigid $\Phi=3$ grains. However, about $30 \%$ of the grains continue to fracture even with $\varepsilon_{f}=200 \%$ for an impact velocity of $\bar{v}_{0}=0.226$ and thus the rise in the transmitted momentum with increasing $\varepsilon_{f}$ is relatively minor for this high impact velocity case.

\subsubsection{Fragmentation from low to high aspect ratio grains}

We now consider the reverse case of low aspect ratio grain fragmentation that results in the grains acquiring a higher aspect ratio after fracture. To achieve this, we consider the case of a slug comprising initially cubic grains with $n=8$ sub-particles. These grains have an initial aspect ratio of unity and comprise 12 connectors between sub-particles as shown in Fig. 10a. Four of these connectors are modelled as beams such that fracture results in the cubic grain breaking into two flake-like grains (Fig. 10b) comprising four sub-particles. The aspect ratio of these flake-shaped grains, as deduced from the best-fit ellipsoid, is 2.41 , i.e. the cubic grain with aspect ratio of unity fractures into flake-like grains with a significantly higher aspect ratio.
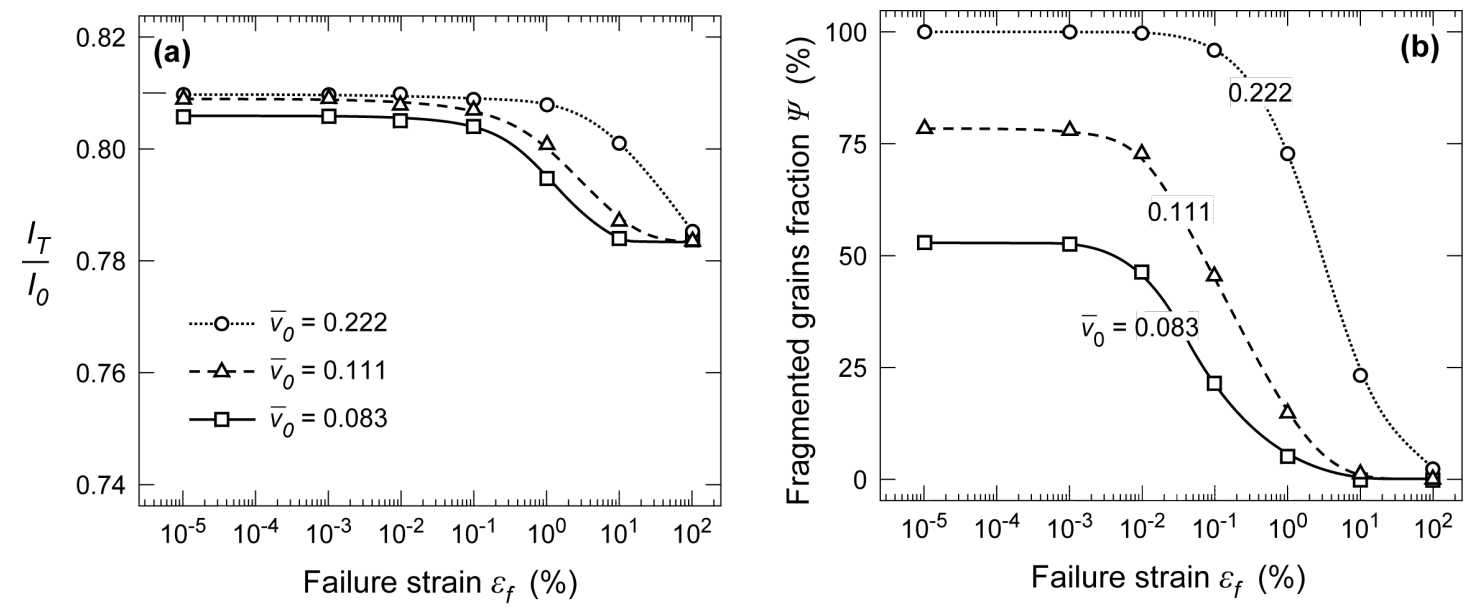

Figure 11: Predictions of (a) the normalised transmitted momentum $I_{T} / I_{0}$ and (b) fraction $\psi$ of the fragmented grains as a function of the failure strain $\varepsilon_{f}$ for slugs comprising cubic $(n=8)$ grains impacting inclined $\left(\alpha=45^{\circ}\right)$ targets. The predictions are shown for $\mu_{T}=1.0$ and three selected values of the normalised impact velocity $\bar{v}_{0}$.

Predictions of $I_{T} / I_{0}$ and $\psi$ versus $\varepsilon_{f}$ are shown in Figs. 11a and $11 \mathrm{~b}$, respectively for three normalised impact velocities $\bar{v}_{0}$. A grain is defined to be fragmented if all the four beam connectors have failed such that the cubic grain has split into the two flake-like grains. In this case grain fragmentation results in the conversion of low aspect ratio grains to high aspect ratio grains and thus sliding of the grains along the target surface becomes more prevalent with increasing levels of fragmentation. As a consequence, $I_{T} / I_{0}$ decreases with decreasing levels of fragmentation (or equivalently increasing $\varepsilon_{f}$ ). This decrease is relatively minor (Fig. 11a) even though the fraction of fractured grains decreases significantly with increasing $\varepsilon_{f}$ (Fig. 
11b). We attribute this to the fact that although the cubic grains have a low aspect ratio, they have a tendency to slide rather than roll and thus transmit a momentum into the inclined target that is comparable to the high aspect ratio rod-shaped grains; see Fig. 8b. Therefore, an increase in aspect ratio of cubic grains due to grain fracture has a relatively minor effect. We speculate that the fracture of initially spherical grains will result in a more significant increase in the transmitted momentum. However, such an analysis is computationally beyond the scope of the current method that uses spherical sub-particles to construct the grains.

We emphasize that while grain fragmentation can result in relatively small changes to the total transmitted momentum, the individual components of the transmitted momentum are more significantly affected. To illustrate this, we include in Figs. 12a and $12 \mathrm{~b}$ predictions of $\left(I_{T}^{Z}-\right.$ $\left.I_{T}^{y}\right) / I_{0}$ for the cases of fragmentation of high aspect ratio grains to low aspect ratio and viceversa, respectively, i.e. the cases in Figs. 9 and 11. As expected, with increasing grain fragmentation (or equivalently decreasing $\left.\varepsilon_{f}\right),\left(I_{T}^{Z}-I_{T}^{y}\right) / I_{0}$ decreases for the case when grains fragment to lower aspect ratios (Fig. 12a) and vice-versa in Fig. 12b. More importantly, grain fragmentation affects $\left(I_{T}^{Z}-I_{T}^{y}\right) / I_{0}$ more substantially as compared to its effect on $I_{T} / I_{0}$.
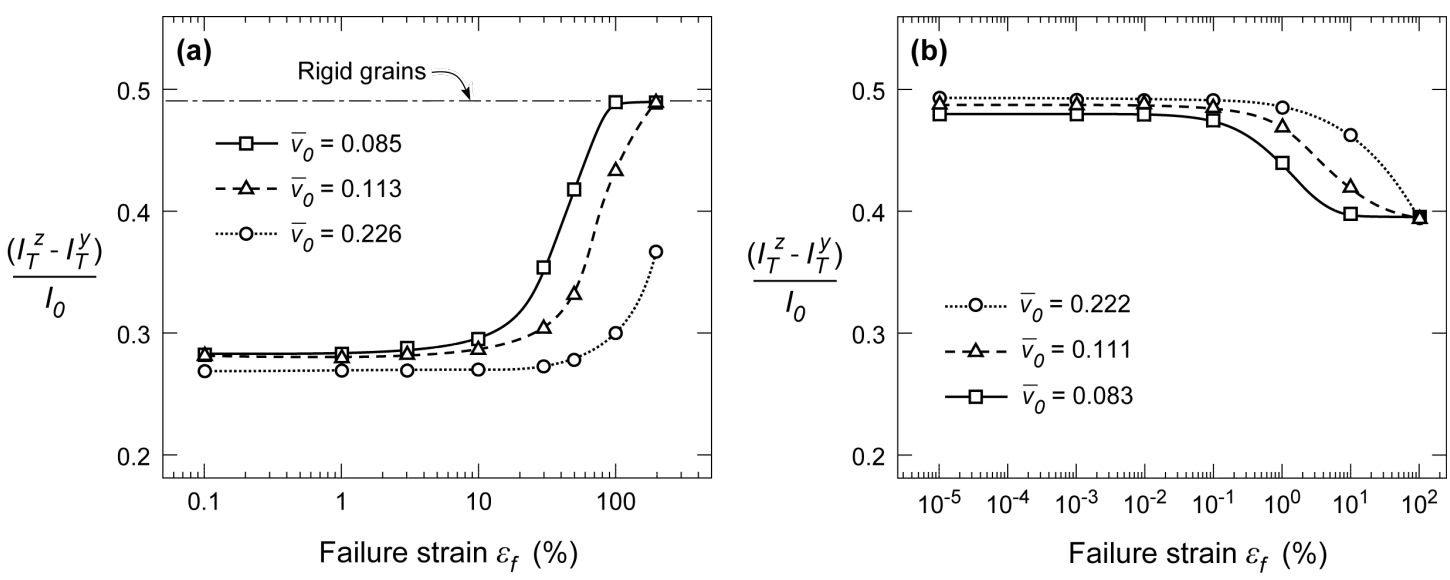

Figure 12: Predictions of $\left(I_{T}^{Z}-I_{T}^{y}\right) / I_{0}$ as a function of the failure strain $\varepsilon_{f}$ for slugs comprising (a) $\Phi=3$ rod-shaped and (b) cubic $(n=8)$ grains impacting inclined $\left(\alpha=45^{\circ}\right)$ targets. The predictions are shown for $\mu_{T}=1.0$ and three selected values of the normalised impact velocity $\bar{v}_{0}$.

Here we have demonstrated that grain fragmentation can influence the momentum transmitted from high velocity granular slugs into targets they impact. While it is attractive to attribute this effect to energy dissipation by the work of fracture associated with grain fracture, there is no direct dependence of the transmitted momentum on energy dissipation. Rather, the grain fracture indirectly affects the transmitted momentum because it changes the shape of the grains which in turn influences the competition between sliding and rolling of the grains on the target surface. This competition affects the transmitted momentum due to frictional interactions between the grains and the target surface. Importantly, in the absence of friction between the grains and the target (i.e. $\mu_{T}=0$ ), grain fracture is predicted to have no influence on the transmitted momentum.

\section{Concluding remarks}

We have reported discrete element calculations to investigate the effect of grain shape and grain fracture on the interaction of high velocity granular media with rigid stationary targets in normal and inclined orientations. The granular media comprised either spherical, rod-shaped 
or cubic grains constructed by combining spherical sub-particles. These sub-particles were connected to each other with either rigid connectors or beam connectors: the beam connectors can fracture so as to simulate fragmentation of the grains.

When grain fracture was suppressed by employing rigid connectors between the sub-particles, the momentum transmitted from the granular slug into normally oriented targets was independent of grain shape due to the symmetry of the problem. However, with increasing angularity of the grains (i.e. rod-shaped or cubic grains compared to spherical grains), the grains tended to slide rather than roll over the target surface. This increased frictional interactions between the grains and the target and resulted in an increase in the momentum transfer into inclined targets. Moreover, it was shown that a simple parameter such as aspect ratio is unable to fully characterise the interaction of the grains with the target surface as the precise grain shape governs the relative propensity of the grains to slide rather than roll on the target surface.

Fracture of grains during the impact event results in dissipation from the work of fracture. However, this work of fracture has no direct effect upon momentum transfer into the targets. Rather, it is the change in the shape of the grains as a result of fracture that directly influences the momentum transmitted into the inclined targets. The numerical calculations presented here show that fracture of rod-shaped grains results in a reduction in the transmitted momentum as the grains break-up into more spherical grains that have a higher propensity to roll rather than slide on the target surface. This reduction increases with decreasing fracture strain of the grains and increasing impact velocity. The situation reverses when the initial shape of the grains was more spherical such that upon fracture the grains become more angular. Field experiments that involve the detonation of buried explosive charges show that soil type and impact velocity influence the fraction of the ejecta momentum that is transmitted into targets. The simulations presented here give some insight into reasons for the observed dependencies although direct experimental quantification of the effect of grain shape and fracture is as yet lacking.

\section{Appendix: Design of the properties of the beam connectors}

The calculations with grain fracture were designed such that they recovered the limit of rigid grains in the absence of beam connector plasticity and failure. To justify the choice of $d_{p} / d_{b}=$ 1 and $\bar{K}_{b}=10$ used in Section 4 , here we consider the case of the $\Phi=3$ rod-shaped grains impacting the inclined target at a velocity $v_{0} / \sqrt{K_{n} /\left(d_{p} \rho_{p}\right)}=0.13$ with $\mu_{T}=1.0$. The beams were assumed to be made from an elastic material (i.e. no plasticity or failure) with $v_{b}=0.17$. Predictions of $\left(I_{T}^{Z}-I_{T}^{y}\right) / I_{0}$ are shown in Fig. Ala as a function of $d_{p} / d_{b}$ for three choices of $\bar{K}_{b}$. The measure of transmitted momentum, $\left(I_{T}^{Z}-I_{T}^{y}\right) / I_{0}$, increases with increasing $\bar{K}_{b}$ and decreasing $d_{p} / d_{b}$. The asymptote corresponding to rigid grains (marked in Fig. Ala) is attained for $d_{p} / d_{b}=1$ and $\bar{K}_{b}=10$. The dependency of $\left(I_{T}^{Z}-I_{T}^{y}\right) / I_{0}$ on $d_{p} / d_{b}$ and $\bar{K}_{b}$ is understood by noting that these grains change shape during the impact event due to the bending of the beams that connect the sub-particles. To illustrate this, we consider the case of a slug with $\Phi=3$ grains constructed by $d_{p} / d_{b}=10$ and $\bar{K}_{b}=1$ beam connectors impacting at $v_{0} / \sqrt{K_{n} /\left(d_{p} \rho_{p}\right)}=0.13$. We then isolate three grains from the slug and follow them through their deformation history as shown in Fig. Alb. At $\bar{t}=0.5$, the beam connectors bend so significantly that the aspect ratio of the grains reduces. This of course promotes rolling of the grains and reduces $\left(I_{T}^{Z}-I_{T}^{y}\right) / I_{0}$. Making the beams stubbier by decreasing $d_{p} / d_{b}$ and increasing their Young's modulus increases the bending stiffness of the beams and makes the 
grains nearly rigid such that the asymptote corresponding to rigid grains is attained for $d_{p} / d_{b}=1$ and $\bar{K}_{b}=10$.
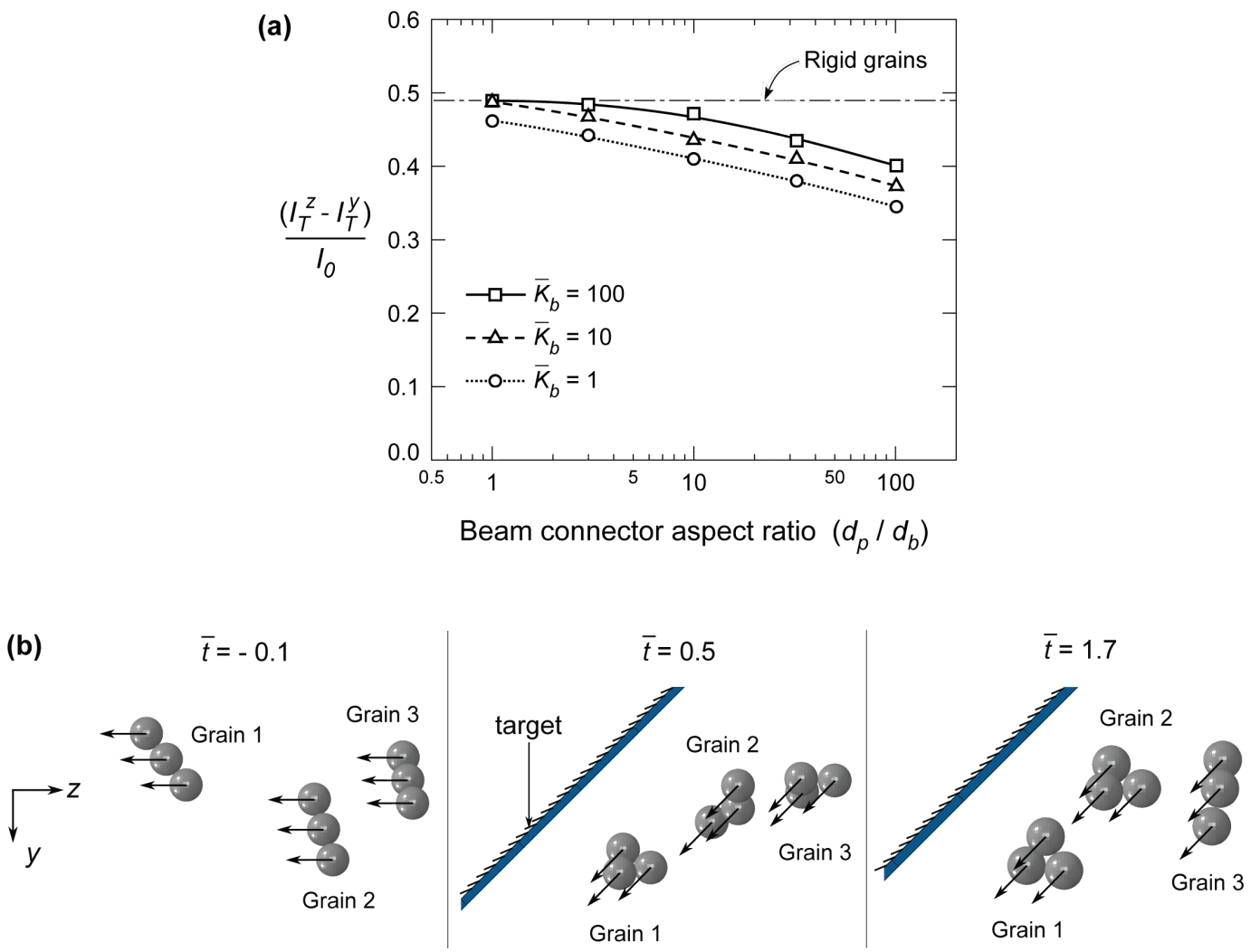

Figure A1: Predictions for slugs comprising $\Phi=3$ rod-shaped grains impacting inclined $\left(\alpha=45^{\circ}\right)$ targets at $v_{0} / \sqrt{K_{n} /\left(d_{p} \rho_{p}\right)}=0.13$ with $\mu_{T}=1.0$. The grains were constructed with elastic beams connecting the sub-particles. (a) The normalised measure of transmitted momentum $\left(I_{T}^{Z}-I_{T}^{y}\right) / I_{0}$ as a function of the beam connector aspect ratio $d_{p} / d_{b}$. Results are shown for 3 choices of the normalised beam axial stiffness $\bar{K}_{b}$ with the asymptote corresponding to rigid grains marked. (b) Snapshots showing the grain shape and velocity directions of three randomly selected grains at three normalised times $\bar{t}$. The beam connectors in this case had $d_{p} / d_{b}=10$ and $\bar{K}_{b}=1$. The normalised time $\bar{t}=0$ corresponds to the instant the slug begins impact with the target.

\section{Acknowledgements}

We are grateful to the Defense Advanced Projects Agency for support of this work under grant number W91CRB-11-1-0005 (Program manager, Dr. J. Goldwasser).

\section{References}

Bathurst, R.J., Rothenburg, L., 1988. Micromechanical aspects of isotropic granular assemblies with linear contact interactions. Journal of Applied Mechanics 55, 17-23.

Behera, B., Kun, F., McNamara, S., Herrmann, H.J., 2005. Fragmentation of a circular disc by impact on a frictionless plate. Journal of Physics: Condensed Matter 17, S2439-S2456. doi: 10.1088/0953-8984/17/24/005 
Bergeron, D., Tremblay, J.E., 2000. Canadian research to characterize mine blast output. Proceedings of the 16th International MABS Symposium, Oxford, UK.

Bergeron, D., Walker, R., Coffey, C., 1998. Detonation of 100-gram anti-personnel mine surrogate charges in sand-A test case for computer code validation. Suffield Report No. 668, Defence Research Establishment Suffield, Ralston, Alberta, Canada.

Borvik, T., Olovsson, L., Hanssen, A.G., Dharmasena, K.P., Hansson, H., Wadley, H.N.G., 2011. A discrete particle approach to simulate the combined effect of blast and sand impact loading of steel plates. Journal of the Mechanics and Physics of Solids 59, 940-958. doi:10.1016/j.jmps.2011.03.004

Cheng, X., Varas, G., Citron, D., Jaeger, H., Nagel, S., 2007. Collective Behavior in a Granular Jet: Emergence of a Liquid with Zero Surface Tension. Physical Review Letters 99, 188001. doi:10.1103/PhysRevLett.99.188001

Cho, G.C., Dodds, J., Santamarina, J.C., 2006. Particle Shape Effects on Packing Density, Stiffness, and Strength: Natural and Crushed Sands. Journal of Geotechnical and Geoenvironmental Engineering 591-602.

Cil, M.B., Alshibli, K.A., 2012. 3D assessment of fracture of sand particles using discrete element method. Geotechnique Letters 161-166.

Clarke, S.D., Fay, S.D., Tyas, A., Warren, J., Rigby, S.E., Elgy, I., Livesey, R., 2014. Repeatability of buried charge testing. In: Proceedings of the 23rd International Symposium on Military Aspects of Blast and Shock. International Symposium on Military Aspects of Blast and Shock, 7-12 September 2014, Oxford, UK.

Cundall, P.A., Strack, O.D.L., 1979. A discrete numerical model for granular assemblies. Géotechnique 29, 47-65. doi:10.1680/geot.1979.29.1.47

Deshpande, V.S., McMeeking, R.M., Wadley, H.N.G., Evans, A.., 2009. Constitutive model for predicting dynamic interactions between soil ejecta and structural panels. Journal of the Mechanics and Physics of Solids 57, 1139-1164. doi:10.1016/j.jmps.2009.05.001

Dharmasena, K.P., Wadley, H.N.G., Liu, T., Deshpande, V.S., 2013. The dynamic response of edge clamped plates loaded by spherically expanding sand shells. International Journal of Impact Engineering 62, 182-195. doi:10.1016/j.ijimpeng.2013.06.012

Ehrgott, J.Q., Akers, S. a., Windham, J.E., Rickman, D.D., Danielson, K.T., 2011. The influence of soil parameters on the impulse and airblast overpressure loading above surface-laid and shallowburied explosives. Shock and Vibration 18, 857-874. doi:10.3233/SAV-2010-0609

Farhadi, S., Behringer, R.P., 2014. Dynamics of Sheared Ellipses and Circular Disks: Effects of Particle Shape. Physical Review Letters 112, 148301. doi:10.1103/PhysRevLett.112.148301

Foedinger, J., 2005. Methodology for improved characterization of landmine explosions, SBIR PhaseII plus program. 2005. Proceedings of the Technical Interchange Meeting, Material Science Corporation.

Fourney, W., Leiste, U., Bonenberger, R., Goodings, D., 2005. Explosive impulse on plates. Fragblast $9,1-17$. 
Goel, A., Uth, T., Wadley, H.N.G., Deshpande, V.S., 2017. Effect of surface properties on momentum transfer to targets impacted by high-velocity sand slugs. International Journal of Impact Engineering 103, 90-106. doi:10.1016/j.ijimpeng.2017.01.001

Grujicic, M., Pandurangan, B., Cheeseman, B.A., 2006. The effect of degree of saturation of sand on detonation phenomena associated with shallow-buried and ground-laid mines. Shock and Vibration 13, 41-61.

Grujicic, M., Pandurangan, B., Mocko, G.M., Hung, S.T., Cheeseman, B.A., Roy, W.N., Skaggs, R.R., 2008a. A Combined Multi-Material Euler/Lagrange Computational Analysis of Blast Loading Resulting from Detonation of Buried Landmines. Multidiscipline Modeling in Materials and Structures 4, 105-124. doi:10.1163/157361108784050086

Grujicic, M., Pandurangan, B., Qiao, R., Cheeseman, B.A., Roy, W.N., Skaggs, R.R., Gupta, R., 2008b. Parameterization of the porous-material model for sand with different levels of water saturation. Soil Dynamics and Earthquake Engineering 28, 20-35. doi:10.1016/j.soildyn.2007.05.001

Hlady, S., 2004. Effect of soil parameters on landmine blast. 18th Military Aspects of Blast and Shock (MABS) Conference, Bad Reichenhall, Germany.

Holloman, R.L., Deshpande, V., Wadley, H.N.G., 2015a. Impulse transfer during sand impact with a solid block. International Journal of Impact Engineering 76, 98-117. doi:10.1016/j.ijimpeng.2014.09.010

Holloman, R.L., Deshpande, V., Wadley, H.N.G., 2015b. Impulse transfer during sand impact with a cellular structure. International Journal of Impact Engineering. doi:10.1016/j.ijimpeng.2015.01.004

Johnson, C.G., Gray, J.M.N.T., 2011. Granular jets and hydraulic jumps on an inclined plane. Journal of Fluid Mechanics 675, 87-116. doi:10.1017/jfm.2011.2

Kyner, A., Dharmasena, K., Williams, K., Deshpande, V., Wadley, H., 2017. High intensity impulsive loading by explosively accelerated granular matter. International Journal of Impact Engineering 108, 229-251. doi:10.1016/j.ijimpeng.2017.02.009

Liu, T., Fleck, N.A., Wadley, H.N.G., Deshpande, V.S., 2013. The impact of sand slugs against beams and plates: Coupled discrete particle/finite element simulations. Journal of the Mechanics and Physics of Solids 61, 1798-1821. doi:10.1016/j.jmps.2013.03.008

NATO: Protection Levels for Occupants of Armoured Vehicles, 3rd Edition, STANAG 4569, 2014.

Neuberger, A., Peles, S., Rittel, D., 2007a. Scaling the response of circular plates subjected to large and close-range spherical explosions. Part II: Buried charges. International Journal of Impact Engineering 34, 874-882. doi:10.1016/j.ijimpeng.2006.04.002

Neuberger, A., Peles, S., Rittel, D., 2007b. Scaling the response of circular plates subjected to large and close-range spherical explosions. Part I: Air-blast loading. International Journal of Impact Engineering 34, 859-873. doi:10.1016/j.ijimpeng.2006.04.001

Parab, N.D., Claus, B., Hudspeth, M.C., Black, J.T., Mondal, A., Sun, J., Fezzaa, K., Xiao, X., Luo, S.N., Chen, W., 2014. Experimental assessment of fracture of individual sand particles at different loading rates. International Journal of Impact Engineering 68, 8-14. doi:10.1016/j.ijimpeng.2014.01.003 
Park, S., Uth, T., Fleck, N.A., Wadley, H.N.G., Deshpande, V.S., 2013. Sand column impact onto a Kolsky pressure bar. International Journal of Impact Engineering 62, 229-242. doi:10.1016/j.ijimpeng.2013.07.003

Pickering, E.G., Chung Kim Yuen, S., Nurick, G.N., Haw, P., 2012. The response of quadrangular plates to buried charges. International Journal of Impact Engineering 49, 103-114. doi:10.1016/j.ijimpeng.2012.05.007

Pingle, S.M., Fleck, N.A., Wadley, H.N.G., Deshpande, V.S., 2012. Discrete element calculations of the impact of a sand column against rigid structures. International Journal of Impact Engineering 45, 74-89. doi:10.1016/j.ijimpeng.2011.10.005

Potapov, A. V, Campbell, C.S., 1997. The two mechanisms of particle impact breakage and the velocity effect. Powder Technology 93, 13-21.

Rigby, S.E., Fay, S.D., Tyas, a., Clarke, S.D., Reay, J.J., Warren, J. a., Gant, M., Elgy, I., 2017. Influence of particle size distribution on the blast pressure profile from explosives buried in saturated soils. Shock Waves. doi:10.1007/s00193-017-0727-7

Rimoli, J.J., Talamini, B., Wetzel, J.J., Dharmasena, K.P., Radovitzky, R., Wadley, H.N.G., 2011. Wetsand impulse loading of metallic plates and corrugated core sandwich panels. International Journal of Impact Engineering 38, 837-848. doi:10.1016/j.ijimpeng.2011.05.010

Samimi, A., Moreno, R., Ghadiri, M., 2004. Analysis of impact damage of agglomerates: Effect of impact angle. Powder Technology 143-144, 97-109. doi:10.1016/j.powtec.2004.04.027

Silbert, L., Ertas, D., Grest, G., Halsey, T., Levine, D., Plimpton, S., 2001. Granular flow down an inclined plane: Bagnold scaling and rheology. Physical Review E 64, 051302. doi:10.1103/PhysRevE.64.051302

Subero, J., Ghadiri, M., 2001. Breakage patterns of agglomerates. Powder Technology 120, 232-243. doi:10.1016/S0032-5910(01)00276-5

Taylor, L.C., Skaggs, R.R., Gault, W., 2005. Vertical impulse measurements of mines buried in saturated sand. Fragblast 9.

Uth, T., Deshpande, V.S., 2014. Response of clamped sandwich beams subjected to high-velocity impact by sand slugs. International Journal of Impact Engineering 69, 165-181. doi:10.1016/j.ijimpeng.2014.02.012

Uth, T., Wadley, H.N.G., Deshpande, V.S., 2015. The effect of inclination and stand-off on the dynamic response of beams impacted by slugs of a granular material. International Journal of Solids and Structures 56-57, 154-174. doi:10.1016/j.ijsolstr.2014.11.019

Wang, Z., Hao, H., Lu, Y., 2004. A three-phase soil model for simulating stress wave propagation due to blast loading. International Journal for Numerical and Analytical Methods in Geomechanics 28, 33-56. doi:10.1002/nag.325

Westine, P.S., Morris, B.L., Cox, P.A., Polch, E., 1985. Development of computer program for floor plate response from land mine explosions. Contract Report No. 1345 for U.S. Army TACOM Research and Development Centre. 
Xie, J., Rittel, D., 2017. Three-dimensional stocastic modelling of metallic surface roughness resulting from pure waterjet peening. International Journal of Engineering Science 120, 241-253.

Zheng, K., Du, C., Li, J., Qiu, B., Fu, L., Dong, J., 2015. Numerical simulation of the impact-breakage behavior of non-spherical agglomerates. Powder Technology 286, 582-591. doi:10.1016/j.powtec.2015.08.041 